\title{
Impact of xylanase expression-inducing compounds on DNA accessibility in Trichoderma reesei
}

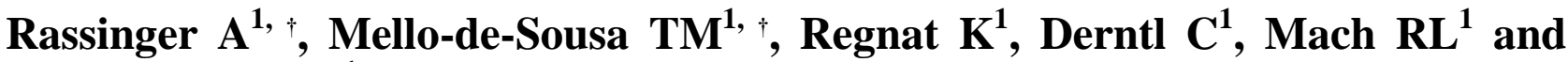 Mach-Aigner $\mathbf{A R}^{1}$}

\begin{abstract}
${ }^{1}$ Institute of Chemical Engineering, Department for Biotechnology and Microbiology, TU Wien, Vienna, Austria. alice.rassinger@tuwien.ac.at, thiago.mello@tuwien.ac.at, katharina.regnat@tuwien.ac.at, christian.derntl@tuwien.ac.at,robert.mach@tuwien.ac.at, astrid.mach-aigner@tuwien.ac.at

${ }^{\dagger}$ Equal contributors
\end{abstract}

Rassinger A, Mello-de-Sousa TM, Regnat K, Derntl C, Mach RL, Mach-Aigner 2016 - Impact of xylanase expression-inducing compounds on DNA accessibility in Trichoderma reesei. Mycosphere 8(3) 432-444, Doi 10.5943/mycosphere/8/3/5

\begin{abstract}
The ascomycete Trichoderma reesei produces industrially applied plant cell wall-degrading enzymes. The two major xylanases XYNI (encoded by xyn1) and XYNII (encoded by xyn2) are amongst those enzymes. The transactivator Xyr1, the repressor Cre1, and gene-specific transcription factors regulate the expression of both xylanases. The inducing compounds D-xylose and sophorose trigger the expression of the xylanases, however, to different extents. D-glucose causes carbon catabolite repression (CCR) mediated by Cre1, which leads to a down-regulation of expression of both. Apart from transcription factors, DNA packaging adds an important layer to the regulation of the production of xylanolytic enzymes. In this study the chromatin status in two upstream regulatory regions (URRs) of the xyn1 and xyn2 genes and the transcript levels were investigated in the wild-type strain QM6a and the hypercellulolytic mutant Rut-C30. This analysis demonstrated more open chromatin and higher transcript levels in both strains and genes under at least one of the two inducing conditions. Additionally, a generally more open chromatin could be observed in Rut-C30 independent of the carbon source that was accompanied by elevated transcript levels. In vivo footprinting analyses demonstrated that protein-DNA interactions differ between Dxylose compared to D-glucose in both strains. In addition, different DNA occupancies were observed in the URR of xyn2 in dependency of the applied inducer. Obviously, the usage of a certain inducer influences the final promoter architecture.
\end{abstract}

Key words - Chromatin - D-xylose - Inducers - sophorose - Trichoderma reesei - xylanases

\section{Introduction}

Trichoderma reesei (teleomorph Hypocrea jecorina (Kuhls et al. 1996)) is a filamentous ascomycete, which exerts a saprotrophic lifestyle. It gains its nutrients by degradation of plant cell wall material. For this purpose, it secretes various cellulases and hemicellulases to break down complex polysaccharides. Synergistic action of $T$. reesei's secreted enzyme cocktail leads to a degradation of the plant biomass.

The two main cellobiohydrolases CBHI (encoded by $c b h 1$ ) and CBHII (encoded by $c b h 2$ ) (Teeri 1983) act on cellulose, the most abundant polysaccharide in nature. However, plant cell 
walls are not only made of cellulose. Hemicelluloses add high variability to the structure of plant cell walls by their side chains. Xylan is the most abundant polysaccharide amongst the hemicelluloses. $T$. reesei secretes two major endo- $\beta-1,4-x y l a n a s e s$ to degrade xylan. These are XYNI (encoded by xyn1) and XYNII (encoded by xyn2) (Törrönen et al. 1992). Apart from their function in nature, endo- $\beta-1,4$-xylanases are widely used in industrial applications (Viikari et al. 1994).

On the transcriptional level, both xylanases share common regulatory mechanisms. Transcriptional regulation is basically dependent on the interplay of the Xylanase regulator 1 (Xyr1) (Stricker et al. 2006) and the carbon catabolite repressor Cre1 (Strauss et al. 1995). Xyr1 is a GAL4-like transactivator and binds to the six nucleotide sequence 5'-GGC(T/A) ) $_{3}$ ' ' (Furukawa et al. 2009). This motif occurs typically as repeats in Xyr1-target promoters. For instance, in the xyn1 promoter, a regulatory element was described to be functional in vivo that contains two Xyr1binding sites arranged as an inverted repeat. Mutation of the Xyr1-binding sites within this element resulted in a loss of xynl expression (Rauscher et al. 2006). Xyrl itself is transcribed at a low basal level on most carbon sources and is induced by the transglycosylation product sophorose (Derntl et al. 2013). In contrast to cellulase-encoding genes, transcription levels of xynl and xyn2 do not respond directly to an increased transcription level of $x y r l$. The authors suggested that the regulation of xylanase expression involves further regulatory mechanisms (Derntl et al. 2013).

Cre 1 is a $\mathrm{C}_{2} \mathrm{H}_{2}$ zinc finger protein, it binds to a 5'-SYGGRG-3' binding motif (Strauss et al. 1995), and it mediates carbon catabolite repression (CCR) in the presence of readily metabolizable carbon sources, such as D-glucose. Cre1 acts directly on the transcription of its target genes, e.g. cbhl (Ilmén et al. 1998), xyn1 (Mach et al. 1996), and xyrl (Mach-Aigner et al. 2008). Consequently, CCR is acting on two different hierarchical levels, i.e. indirectly, by repressing the expression of the main activator Xyr1, and directly, by binding to the upstream regulatory regions (URRs) of the respective target genes. As a third common transcription factor, the CCAAT-box binding protein complex Hap2/3/5 plays a role in the expression of xyn1 and xyn2 (Würleitner et al. 2003, Rauscher et al. 2006).

In addition, gene-specific transcription factors are involved in regulation of $x y n 1$ and/or xyn 2 transcription. For instance, the Activator of cellulases 1 (Ace1) (Aro et al. 2003) acts as a repressor of xynl expression (Rauscher et al. 2006). The Activator of cellulases 2 (Ace2) (Aro et al. 2001) and the Xylanase promoter-binding protein 1 (Xpp1) (Mach-Aigner et al. 2010, Derntl et al. 2015 ) influence xyn2 transcription (Würleitner et al. 2003, Stricker et al. 2008a, Derntl et al. 2015). Xpp1 exerts a repressive function in regard to transcriptional regulation, whereas Ace2 rather acts as an activator. Although both xylanase-encoding genes share common transcription factors, their regulation of gene expression varies in terms of their response to available carbohydrates. For $x y n 2$, a low basal transcription level is observed even under repressing conditions (e.g. on D-glucose) (Derntl et al. 2013). Induction of xyn2 expression occurs on sophorose, xylobiose, and in the presence of D-xylose (Zeilinger et al. 1996, Würleitner et al. 2003). On the other hand, xynl is subjected to CCR and is therefore completely shut off under repressing conditions (Mach et al. 1996). Induction of xynl expression is mainly achieved on D-xylose (Zeilinger et al. 1996). The reasons for the different induction behaviour of the two xylanases are not fully understood yet.

Additionally to transcription factors, chromatin contributes to the regulation of transcription. In a recent study, it was shown that chromatin packaging plays a role in the regulation of transcription of the cellulase-encoding genes cbhl and $c b h 2$ (Ries et al. 2014, Mello-de-Sousa et al. 2016). Further, in an earlier study, it was reported that in the absence of Xyr1, the chromatin status of cellulase-encoding genes got denser and was accompanied by a decreased expression (Mello-de-Sousa et al. 2015). When the cellulase expression and the chromatin status are pulled together, the industrially important strain Rut-C30 has to be mentioned. Rut-C30 was generated by 3 mutagenesis steps starting from QM6a as the originate strain (Montenecourt \& Eveleigh 1979a, Montenecourt \& Eveleigh 1979b). This yielded the high cellulase-producing strain with a phenotype released from CCR. Importantly, Rut-C30 bears a truncated version of Cre1 (Cre1-96), which seems to contribute to a more open chromatin status (Mello-de-Sousa et al. 2014). Moreover, 
MNaseI digestion of the $c b h 1$ and $c b h 2$ promoter were performed. It was found that the structural gene of cbhl shows a complete loss of positioned nucleosomes in Rut-C30 despite the tested carbon source (Ries et al. 2014). Whereas in $c b h 2$ both, structural gene and the region located upstream of the start codon showed a full depletion of nucleosomes under repression and inducing conditions (Zeilinger et al. 2003). In terms of xylanase production, Rut-C30 is also relevant for industrial purposes considering the release of $x y n 1$ expression from CCR. The extent of inducibility of xyn1 and xyn 2 expression was found to be different on D-xylose compared to sophorose in RutC30 (Derntl et al. 2013). However, a possible influence of the chromatin status on the expression of xylanases has not been investigated yet (as it was done for the cellulases).

In this study, we investigated the chromatin status and the protein-DNA interactions in the URRs of both xylanase-encoding genes. A special focus was the comparative analysis of the two different inducing conditions (D-xylose, sophorose) in relation to a repressing (D-glucose) condition. To this end, CHART-PCR, qPCR, and in vivo footprinting analyses were performed to investigate two selected regions in the URR of each xylanase-encoding gene in the wild-type strain QM6a and in Rut-C30.

\section{Materials \& methods}

\section{Fungal strains}

The following T. reesei strains were used throughout this study: T.reesei QM6a (ATCC 13631) is the wild-type strain and is referred to as reference strain. The other strain of interest is the hypercellulolytic strain Rut-C30 (ATCC 56765), which is the common ancestor of most T.reesei industrial strains. All strains were maintained on $3 \%$ malt extract agar plates containing $0.1 \%$ $(\mathrm{w} / \mathrm{v})$ peptone.

\section{Growth conditions}

For carbon source replacement experiments, fungal mycelia were pre-cultured in 11 Erlenmeyer flasks on a rotary shaker $(180 \mathrm{rpm})$ at $30{ }^{\circ} \mathrm{C}$ for $24 \mathrm{~h}$ in $250 \mathrm{ml}$ of Mandels-Andreotti (MA) medium (Mandels 1985) containing $1 \%(\mathrm{w} / \mathrm{v})$ glycerol as sole carbon source. For inoculation $10^{9}$ conidia per litre (final concentration) were used. Pre-grown mycelia were washed, equally distributed, and resuspended in $20 \mathrm{ml}$ or $100 \mathrm{ml}$ MA media containing $1 \%$ (w/v) Dglucose, $0.5 \mathrm{mM}$ D-xylose, $2 \mathrm{mM}$ sophorose or no carbon source, respectively. Replacement cultures were then incubated on a rotary shaker $(180 \mathrm{rpm})$ at $30{ }^{\circ} \mathrm{C}$ for $3 \mathrm{~h}$. Samples were derived from three biological replicates and were pooled before RNA extraction and chromatin digestion.

\section{Analysis of transcript levels}

$0.01-0.03 \mathrm{mg}$ frozen fungal mycelia were homogenized in $1 \mathrm{ml}$ of peqGOLDTriFast DNA/RNA/protein purification system reagent (PEQLAB Biotechnology, Erlangen, Germany) using a FastPrep(R)-24 cell disrupter (MP Biomedicals, Santa Ana, CA, USA). RNA isolation was conducted following the manufacturer's instructions and the concentration was measured using the NanoDrop 1000 (Thermo Scientific, Waltham, US). Synthesis of cDNA from mRNA was carried out using the RevertAidTM H Minus First Strand cDNA Synthesis Kit (Thermo Scientific, Waltham, USA) according to the manufacturer's instructions. Quantitative, reverse transcription PCRs (RT-qPCRs) were performed in a Rotor-Gene Q system (Qiagen, Hilden, Germany). All reactions were performed in triplicates. The reaction mixture had a final volume of $15 \mu \mathrm{l}$ containing $7.5 \mu 12 \times$ iQ SYBR Green Mix (Bio-Rad, Hercules, USA), $100 \mathrm{nM}$ forward and reverse primer, and $2.5 \mu \mathrm{l}$ cDNA (diluted 1:20). Primer sequences are provided in table 1. Cycling conditions and control reactions were performed as described previously (Steiger et al. 2010). Data normalization using sarl and act as reference genes, and calculations were performed as published previously (Steiger et al. 2010). 


\section{Chromatin accessibility real-time PCR (CHART-PCR)}

DNaseI digestions of chromatin and subsequent quantitative PCR (qPCR) analyses were carried out as described before (Mello-de-Sousa et al. 2014). qPCR analyses of the DNaseI-treated samples were performed to measure the relative abundance of DNA of the target regions. PCRs were performed in triplicates in a Rotor-Gene Q system (Qiagen) using the reaction mixture (final volume $20 \mu \mathrm{l}$ ) and the cycling conditions as described before (Mello-de-Sousa et al. 2014). Primer sequences are provided in table 1.

Table 1 Primer sequences and their employment in the study are listed here. All sequences are given in 5' to 3' orientation.

\begin{tabular}{|c|c|c|}
\hline Primer name & Sequence & Employment \\
\hline epixyn1_1Tr_f & GCACTCCAAGGCCTTCTCCTGTACT & $\begin{array}{l}\text { xynl CHART, region }-577 \text { to } \\
-278\end{array}$ \\
\hline epixyn1_1Tr_r & TAGATTGAACGCCACCCGCAATATC & \\
\hline epixyn1_3Tr_f & GTCGATATTGCGGGTGGCGTTCAAT & $\begin{array}{l}\text { xynl CHART, region }-306 \text { to } \\
-10\end{array}$ \\
\hline epixyn1_3Tr_r & TTTGTGCGTGTTTTCCTTGAAGTCG & \\
\hline epixyn2_1Tr_f & GTGCCGATGAGACGCTGCTGAGAAA & $\begin{array}{l}\text { xyn2 CHART, region }-527 \text { to } \\
-252\end{array}$ \\
\hline epixyn2_1Tr_r & GATATTGCGCCTTGCAACACCATCG & \\
\hline epixyn2_2Tr_f & CTCGAGACGGCTGAGACAGCAGCAT & $\begin{array}{l}x y n 2 \mathrm{CHART} \text {, region }-311 \text { to } \\
-38\end{array}$ \\
\hline epixyn2_2Tr_r & TGTCTTTTGGGCTTGGAGGGGTTGT & \\
\hline actfw & TGAGAGCGGTGGTATCCACG & RT-qPCR \\
\hline actrev & GGTACCACCAGACATGACAATGTTG & \\
\hline sar1fw & TGGATCGTCAACTGGTTCTACGA & \\
\hline sar1rev & GCATGTGTAGCAACGTGGTCTTT & \\
\hline xyn1f & CAGCTATTCGCCTTCCAACAC & \\
\hline xyn1r & CAAAGTTGATGGGAGCAGAAG & \\
\hline $\operatorname{taqxyn} 2 \mathrm{f}$ & GGTCCAACTCGGGCAACTTT & \\
\hline $\operatorname{taqxyn} 2 \mathrm{r}$ & CCGAGAAGTTGATGACCTTGTTC & \\
\hline xyn1_1 oligo $3 \mathrm{f}$ & [6-FAM]AGCCCCAGCAGAACATGTCGTCGG & $\begin{array}{l}\text { xyn1 footprint, region } 1-869 \text { to } \\
-598\end{array}$ \\
\hline xyn1_1 oligo $3 \mathrm{r}$ & [6-FAM] AGGGGCTTCATGTCGGACTTGCGG & \\
\hline xyn1_2 oligo $3 \mathrm{f}$ & $\begin{array}{l}\text { [6-FAM] } \\
\text { AGCAGCTACATCTACCAAGACACTCGTGC } \\
\text { A }\end{array}$ & $\begin{array}{l}\text { xynl footprint, region } 2-513 \text { to } \\
-361\end{array}$ \\
\hline RG72 & $\begin{array}{l}\text { [6-FAM] } \\
\text { GAGGTTGAAAGCGGCTCGTACAGTATCC }\end{array}$ & \\
\hline xyn2_1 oligo $3 \mathrm{f}$ & $\begin{array}{l}\text { [6-FAM }] \\
\text { TGTGATGCTGCTGCTGATGGCTAATCCC }\end{array}$ & $\begin{array}{l}\text { xyn2 footprint, region } 1-580 \text { to } \\
-373\end{array}$ \\
\hline xyn2_1 oligo $3 \mathrm{r}$ & $\begin{array}{l}\text { [6-FAM] } \\
\text { CTCATCAAGCTTGCCTCGTCTCCGC }\end{array}$ & \\
\hline RG131 & $\begin{array}{l}{[6-\mathrm{FAM}]} \\
\text { CCGTTATTCAGACAATGTATGTGCCGGGC }\end{array}$ & $\begin{array}{l}\text { xyn2 footprint, region } 2-258 \text { to } \\
-73\end{array}$ \\
\hline RG132 & $\begin{array}{l}\text { [6-FAM] } \\
\text { GTTGTTGTGTCTTTTGGGCTTGGAGGGG }\end{array}$ & \\
\hline
\end{tabular}

The amount of intact input DNA of each sample was calculated by comparing the threshold values of the PCR amplification plots with a standard curve generated for each primer set using serial dilutions of genomic, undigested DNA. The chromatin accessibility index (CAI) was defined as: $\mathrm{CAI}=(\mathrm{Dc} 1+\mathrm{Dc} 2) / 2 \mathrm{Ds}$, where Ds is the amount of intact DNA detected for each target region, and Dc1 and Dc2 are the amounts of intact DNA detected for the promoter regions of sar1 and act, respectively, which were used as reference genes for normalization. 


\section{In vivo footprinting analysis}

In vivo methylation using dimethyl sulphate (DMS) followed by ligation-mediated PCR was performed as described previously (Gorsche et al. 2013). Primer sequences are provided in table 1. The separation of fragments was performed by capillary gel electrophoresis (Microsynth, Balgach, Switzerland) and results were analysed using the ivFAST program (Gorsche et al. 2013). The ivFAST output data is statistically tested (Student's t-test) within a two-sided $95 \%$ confidence interval and gives significantly different values. Landscape visualization of data was generated as described previously (Mello-de-Sousa et al. 2014) and displayed as the protein-DNA interaction index (PDI). The PDI indicates a relative ratio of protein-DNA interactions derived from mycelia incubated on D-xylose or sophorose compared to D-glucose (reference condition) for both strains and regions.

\section{Results and Discussion}

\section{Other than by D-xylose, the induction by sophorose always goes along with chromatin opening}

To investigate the role of the chromatin status during the induction of the xylanase expression, the wild-type strain QM6a and the strain Rut-C30 (released from CCR) were pre-grown on glycerol and then transferred to the two inducing substances, namely D-xylose and sophorose. Further, D-glucose was used as a repressing carbon source and no carbon source as the reference condition. The fungal mycelia were then subjected to DNaseI digestions and a CHART-PCR assay was employed to determine the chromatin accessibility of the URRs of xyn 1 and xyn2. To relate the chromatin accessibility to the transcription profile of both strains, RT-qPCRs of the respective genes (i.e. xynl and xyn2) were performed. Two regions for the CHART-PCR of each gene were chosen based on the frequency of occurrence of transcription factor-binding sites in the respective URRs. The chosen regions are depicted in figure 1.

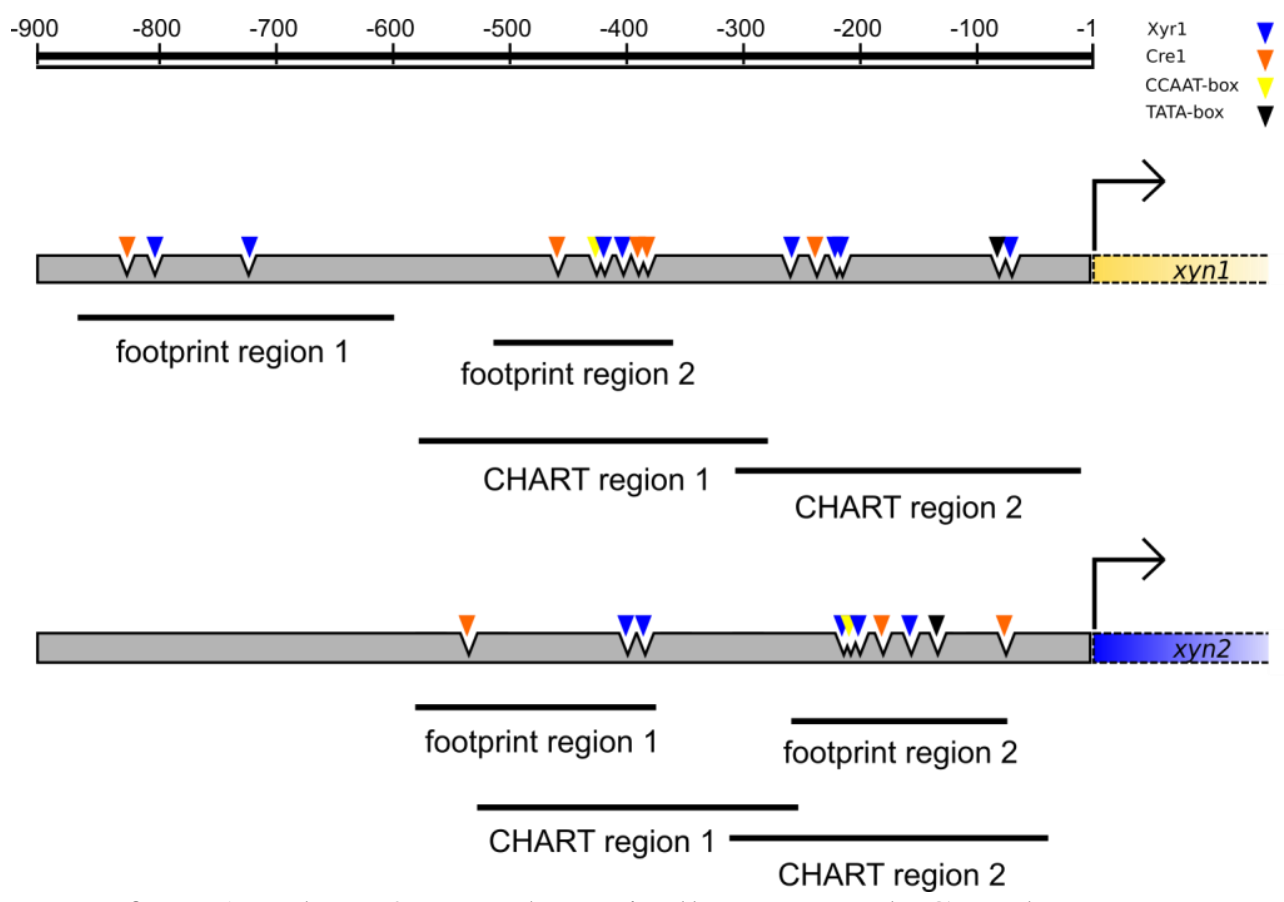

Fig 1 - URRs of xyn1 and xyn2 are schematically presented. Grey bars represent URRs of the respective genes. Yellow (xyn1) and blue (xyn2) bars depict the structural genes. Within the URRs, transcription factor-binding sites are symbolized by coloured triangles: Xyr1-binding site 5'GGC(T/A) 3-3' (blue), Cre1-binding site 5'-SYGGRG-3' (orange), CCAAT-box (yellow), and TATA-box (black). Positions are indicated by the scale on top. Regions for CHART-PCR and in vivo footprinting analyses are marked by black bars and were termed CHART region 1 and 2, or footprint region 1 and 2 , respectively. 
In the case of $x y n 1$, the CHART region 1 comprises a URR that was proven to be functional, whereas the CHART region 2 bears the TATA-box and putative transcription factorbinding sites in close proximity. The cis elements in the URR of $x y n 1$, which were previously identified as functional in vivo, are a CCAAT-box (position -428), two Xyr1-binding sites (positions -404 and -420), and a double Cre1-binding site (positions -383 and -391) (Rauscher et al. 2006). In the case of xyn2, the CHART region 1 contains putative cis elements located further upstream. The CHART region 2 is located near the TATA-box and bears a previously described regulatory element of xyn2. The following cis elements belong to this region: a CCAAT-box (position -216) that is surrounded by two Xyr1-binding sites (positions -208 and -222), and one Cre1-binding site (position -188) (Würleitner et al. 2003, Stricker et al. 2008b).
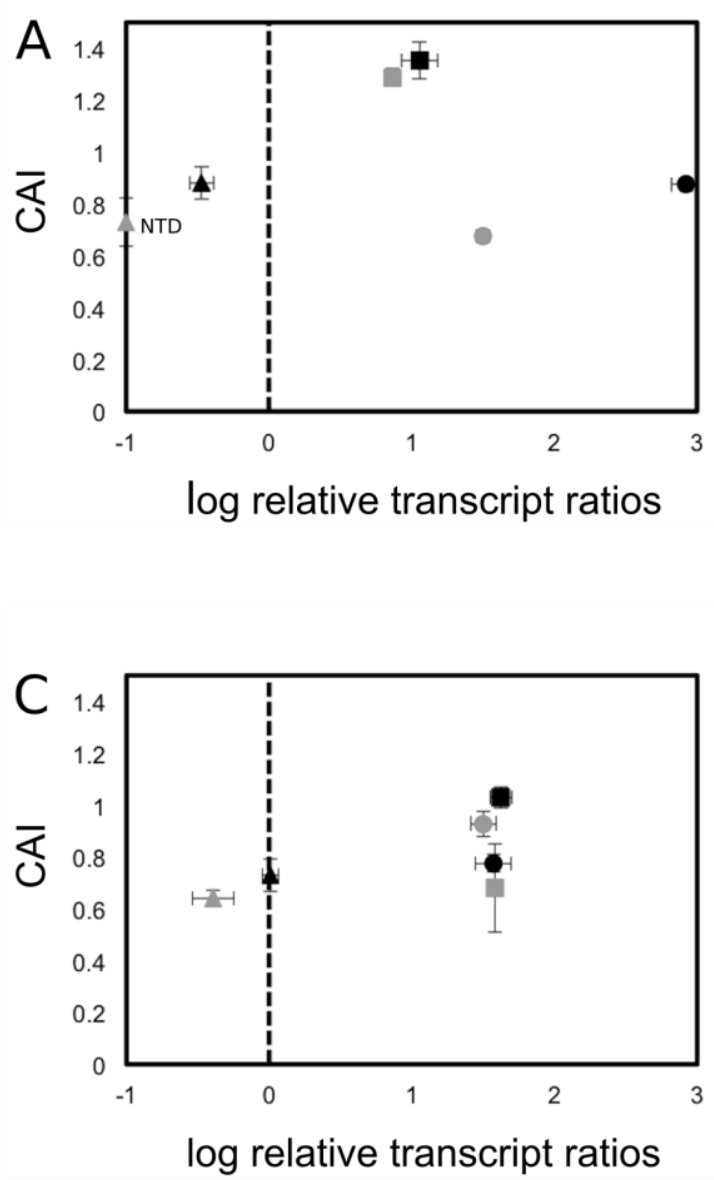

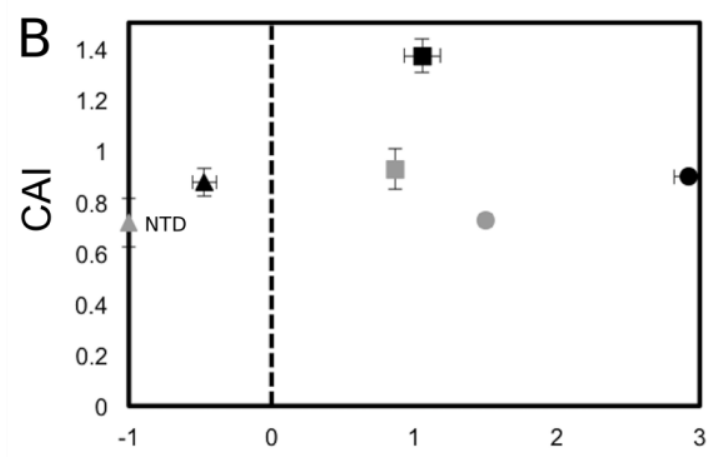

log relative transcript ratios

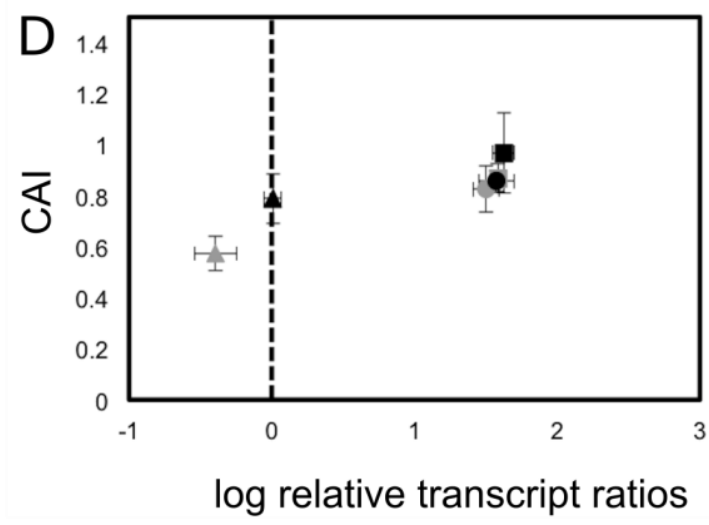

Fig 2 A-D - The chromatin status and the transcript levels were assessed by CHART-PCR analyses and RT-qPCR in the wild-type strain QM6a (grey) and in Rut-C30 (black). Both strains were pregrown on glycerol and incubated for $3 \mathrm{~h}$ on D-glucose (triangles), D-xylose (circles), sophorose (squares), and no carbon source, which was used as the reference condition for transcript analysis (indicated by the dashed vertical lines). The relative transcript ratios are displayed in logarithmic scale on the $\mathrm{x}$-axis and the chromatin accessibility indices (CAIs) are depicted on the y-axis. The transcript levels and the CAIs are normalized to act and sarl. NTD means "no transcript detected" and was set to -1 . The results for the investigated CHART regions $1(\mathrm{~A}, \mathrm{C})$ and $2(\mathrm{~B}, \mathrm{D})$ are provided for $x y n 1$ (A, B) and xyn2 (C, D). 
In the case of $x y n 1$, the chromatin status does not change on D-xylose in comparison to Dglucose in both CHART regions and strains (Fig. 2 A and B), but the transcript levels are considerably induced by D-xylose in both strains (Fig. 2 A or B). In Rut-C30, the extent of induction by D-xylose is even higher than in QM6a. The chromatin is slightly more open in RutC30 than in QM6a, which is a general trend also on the other investigated carbon sources (Fig. 2 A and B). The chromatin reacts differently on sophorose compared to D-xylose. A chromatin opening can be observed in the presence of sophorose compared to D-glucose for both strains and regions (Fig. 2 A and B). However, the extent of induction on sophorose was lower than on D-xylose in both strains. Altogether, an opening of chromatin did accompany the induction achieved with sophorose, but no opening was associated with an even more pronounced induction by $\mathrm{D}$-xylose (Fig. 2 A and B).

In contrast to xyn1, an opening effect of the chromatin is observed on D-xylose compared to D-glucose in both investigated CHART regions of xyn2 in QM6a (Fig. 2 C and D). Although, this opening did not occur in Rut-C30, xyn2 gene expression is induced in both strains on D-xylose. The chromatin opening on D-xylose together with an increased transcript level was found in QM6a only (Fig. $2 \mathrm{C}$ and D). Sophorose induces xyn2 expression to a similar extent as D-xylose in both strains (Fig. $2 \mathrm{C}$ and D). In the case of sophorose, the chromatin becomes more accessible compared to Dglucose in one of the investigated regions in each strain (CHART region 2 in QM6a, CHART region 1 in Rut-C30). Summarizing, the achieved induction (regardless of the inducing compound) was accompanied by an opening of chromatin in the wild-type strain, and a sophorose-specific opening in Rut-C30.

\section{D-xylose changes protein-DNA interaction in the wild-type strain, but hardly in Rut-C30}

In the course of transcription analyses, the expression of both xylanases was found to be induced by D-xylose in both strains (Fig. 2 A-D). However, the expression of $x y n 1$ can be induced stronger in Rut-C30 than in QM6a (Fig. 2 A and B). Interestingly, an opening of the chromatin didn't accompany the induction of xynl expression by D-xylose in either strain, whereas it did for the induction of xyn2 expression (Fig. $2 \mathrm{C}$ and D). To study the strain-specific differences during induction of xylanase expression in detail, in vivo footprinting analyses were performed. Therefore, both strains were pre-grown on glycerol and transferred to D-glucose and D-xylose. A DMS treatment allowed us to capture in vivo DNA occupancy by proteins within two footprinting regions that were chosen based on the frequency of occurrence of transcription factor-binding sites and regulatory elements (Fig. 1).

We could detect strong differences of the protein-DNA interactions comparing D-xylose to D-glucose in the wild-type strain in the region 1 of the $x y n 1$ URR (-869 to -598) (Fig. 3 A). In RutC30, though, the differences in DNA occupancy between the two carbon sources were not as pronounced as in QM6a (Fig. 3 A). In comparison to region 1, the region 2 of the xyn1 URR (positions -513 to -361) shows a different in vivo footprinting pattern. Less pronounced differences (concerning intensity and frequency) in protein-DNA interactions on D-xylose compared to Dglucose were detected in both strains (Fig. 3 B). In QM6a, the differences in protein-DNA interactions were mainly found on essential cis acting elements. In Rut-C30, the differences of DNA occupancies between D-xylose and D-glucose were detected in close proximity to one Xyr1binding site (position -426) and to the Cre1-binding sites (-383, -391 and -466) (Fig. 3 B). 

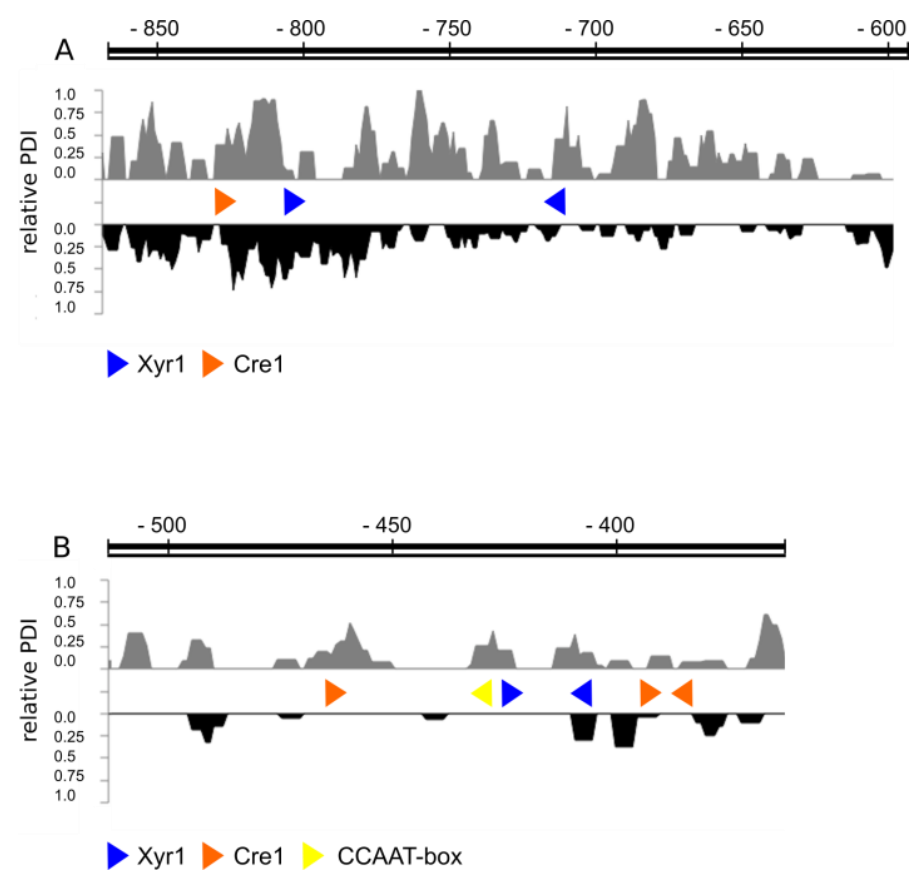

Fig. 3 A-B - In vivo footprinting landscapes of the xynl URR are presented for footprinting regions 1 (A) and 2 (B). Fungal mycelia were pre-grown and transferred to D-xylose (inducing) and D-glucose (repressing). The chromosomal DNA was methylated in vivo by DMS. The profiles of the relative protein-DNA interaction indices (PDIs) of the wildtype strain QM6a (grey) and Rut-C30 (black) are depicted on the y-axis. The PDIs indicate differences in protein-DNA interactions on D-xylose compared to D-glucose for each strain and region. Putative transcription factor-binding sites within the URR (x-axis) are symbolized by coloured triangles: Xyr1 (blue), Cre1 (orange), and CCAAT-box (yellow). Positions are indicated by the scale on top.
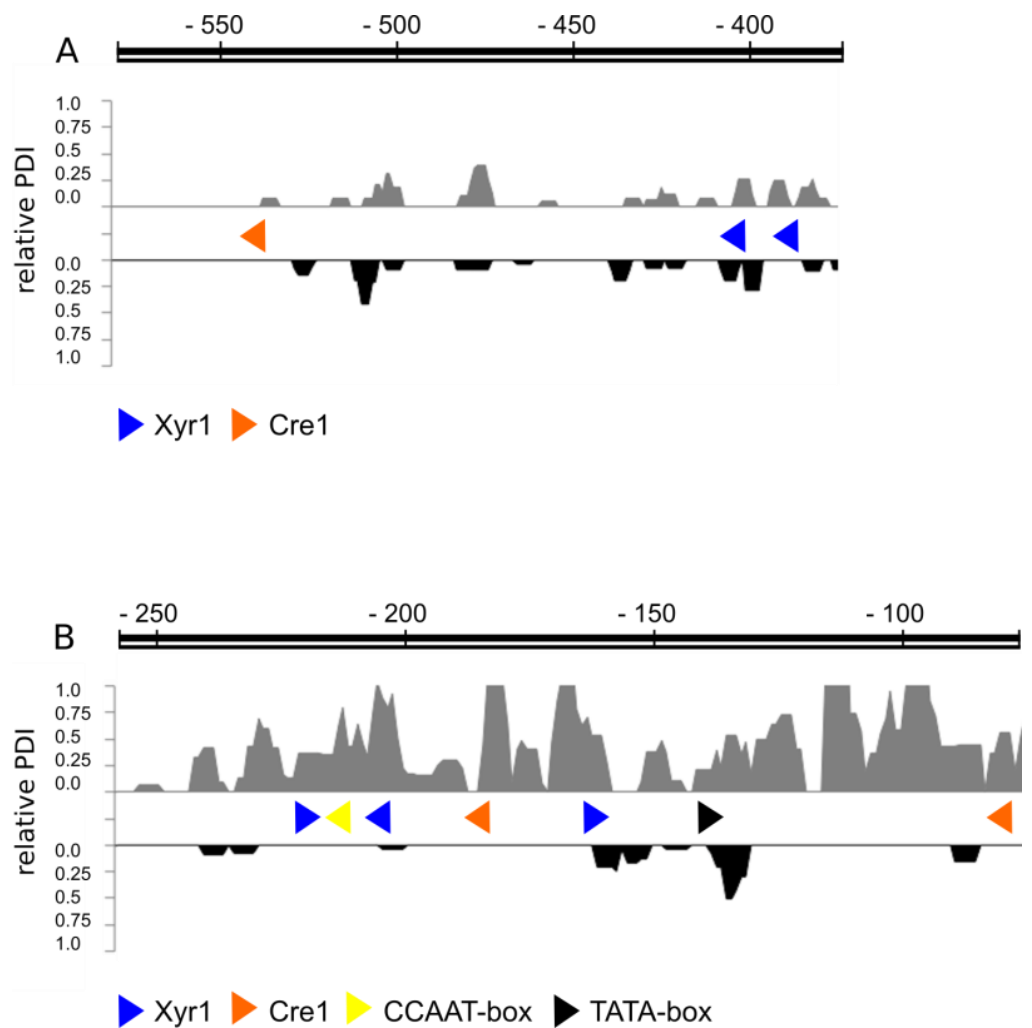

Fig 4 A-B - In vivo footprinting landscapes of the xyn2 URR are presented for footprinting regions 1 (A) and 2 (B). Fungal mycelia were pre-grown and transferred to D-xylose (inducing) and D-glucose (repressing). The chromosomal DNA was methylated in vivo by DMS. The profiles of the relative protein-DNA interaction indices (PDIs) of the wildtype strain QM6a (grey) and Rut-C30 (black) are depicted on the y-axis. The PDIs indicate differences in protein-DNA interactions on D-xylose compared to D-glucose for each strain and region. Putative transcription factor-binding sites within the URR (x-axis) are symbolized by coloured triangles: Xyr1 (blue), Cre1 (orange), the CCAAT-box (yellow), and the TATA-box (black). Positions are indicated by the scale on top. 
In the case of $x y n 2$, we obtained for region 1 (positions -580 to -373) a similar pattern for both strains, i.e. we detected only a few changes in protein-DNA interactions on D-xylose compared to D-glucose (Fig. 4 A). However, in region 2 (positions -258 to -73) strong and frequently occurring differences in protein-DNA interactions between D-xylose and D-glucose were detected in the case of QM6a (Fig. 4 B). In contrast, the in vivo footprinting pattern in RutC30 shows again hardly any differences in DNA occupancy on D-xylose compared to D-glucose. Notably, the detected differences were found on and in close proximity to the TATA-box (Fig. 4 B).

Summarizing, for QM6a we found that in both genes (i.e. xyn1 and xyn2) at least one region exhibits pronounced differences in protein-DNA interaction in response to D-xylose compared to D-glucose. In contrary, in Rut-C30, the DNA occupancy hardly changed in the presence of Dxylose compared to D-glucose. It is important to note that Rut-C30 had a slightly more open chromatin than QM6a and a better induction of xyn1 expression triggered by D-xylose (compare Fig. 2 A and B). Considering all these observations, we hypothesize that the transcription machinery can access the generally more open chromatin in Rut-C30 more easily, resulting in a higher inducibility of the gene expression. However, neither the open chromatin status, nor the induction seems to be influenced by regulatory proteins binding specifically in response to the presence of D-xylose or D-glucose in Rut-C30 according to the in vivo footprinting analyses. A possible explanation for the lack of differences in the protein-DNA interaction between D-xylose and D-glucose might be the presence of a truncated version of Cre1, i.e. Cre1-96 (Mello-de-Sousa et al. 2014). It was reported that Cre1-96 is involved in the open chromatin status of the promoter regions of the cellulase-encoding genes $c b h 1$ and $c b h 2$. Functional Cre1-sites were found to be occupied in these URRs under both, inducing and repressing conditions. Therefore, Mello-de-Sousa and co-workers suggested that Cre1-96 no longer binds DNA in a D-glucose-specific manner (Mello-de-Sousa et al. 2014). Consequently, we assume that Cre1-96 also binds on the investigated xyn1 and xyn2 URRs under both conditions, thereby contributing to the reduced differences observed in the in vivo footprinting analyses. Further, we suggest that the carbohydrate-independent binding of Cre1-96 is related to the more open chromatin in Rut-C30 detected under all conditions tested. However, this model does not explain the strong differences in induction between D-xylose and D-glucose of the xynl and xyn2 gene expression in Rut-C30. In this regard, the in vivo footprinting analyses point towards the TATA-box. The detected differences of DNA occupancies directly at the TATA-box in Rut-C30 suggest a hot spot of D-xylose-dependent interactions there, such as the assembly of the transcription machinery and/or recruiting proteins.

\section{Protein-DNA interaction pattern is similar in the wild-type strain and Rut-C30 when applying the inducer sophorose}

In the case of $x y n 2$, a similar intensity of induction was achieved by sophorose as by Dxylose (Fig. $2 \mathrm{C}$ or D). Therefore, we became interested in studying protein-DNA interactions on the $x y n 2$ URR under sophorose-induced conditions in an analogous experiment as performed for Dxylose. We conducted an in vivo footprinting analysis of mycelia samples incubated on sophorose and compared them to samples derived from incubation on D-glucose.

The in vivo footprinting analysis of region 1 resulted in rather small and regularly distributed differences comparing sophorose and D-glucose (Fig. 5 A). A clear cis element-specific pattern could not be observed for either strain, similarly to the results for the comparison of Dxylose to D-glucose (compare Fig. 5 A to Fig. 4 A).

As mentioned, strong differences in protein-DNA interactions were found across the region 2 on D-xylose compared to D-glucose in QM6a (Fig. 4 B). Using sophorose as an inducing substance, only minor differences in protein-DNA interactions were detected compared to Dglucose, and even a complete loss of any interaction differences directly at the TATA-box was observed (Fig. 5 B). The Xyr1-binding sites remained occupied using sophorose as inducer in the wild-type strain, however, the differences to D-glucose were not as strong as on D-xylose (compare Fig. 5 B to Fig. 4 B). Interestingly, in Rut-C30, the in vivo footprinting pattern resulting from 
comparison of both inducers to D-glucose showed hardly any differences (compare Figs. 4 and 5 B). However, also in Rut-C30 a reduction in the intensity was found around the TATA-box when sophorose was used as inducer (Fig. 5 B) instead of D-xylose (Fig. 4 B).
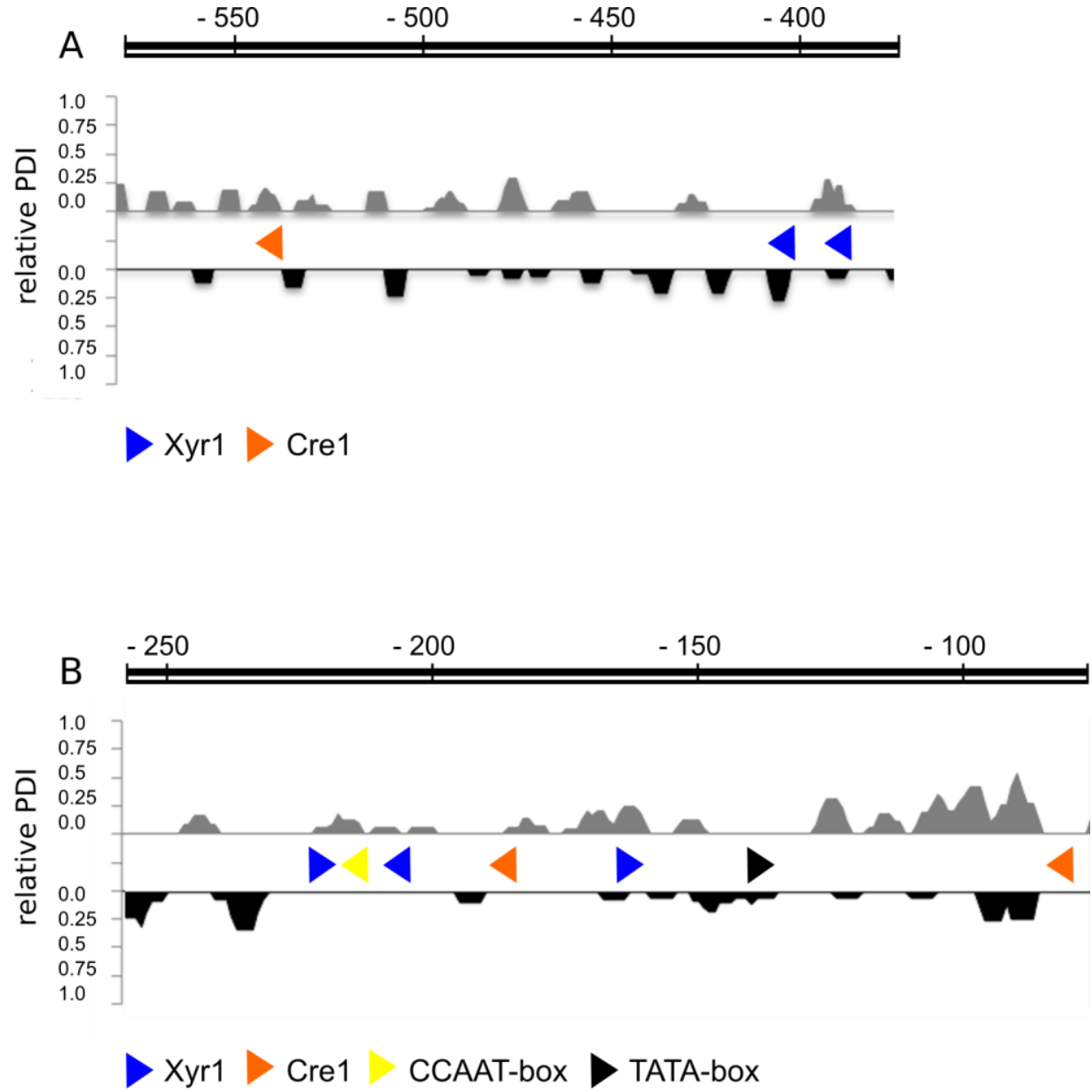

Fig 5 A-B - In vivo footprinting landscapes of the $x y n 2$ URR are presented for footprinting regions 1 (A) and 2 (B). Fungal mycelia were pre-grown and transferred to sophorose (inducing) and Dglucose (repressing). The chromosomal DNA was methylated in vivo by DMS. The profiles of the relative protein-DNA interaction indices (PDIs) of the wild-type strain QM6a (grey) and Rut-C30 (black) are depicted on the y-axis. The PDIs indicate differences in protein-DNA interactions on sophorose compared to D-glucose for each strain and region. Putative transcription factor-binding sites within the URR (x-axis) are symbolized by coloured triangles: Xyr1 (blue), Cre1 (orange), the CCAAT-box (yellow), and the TATA-box (black). Positions are indicated by the scale on top.

Altogether, it became apparent that the interplay of proteins contacting the DNA leads to a different pattern for sophorose and D-xylose on the investigated region 2. Notably, this region bears the cis acting elements that were earlier described as functional for the regulation of $x y n 2$ expression (Würleitner et al. 2003, Stricker et al. 2008b). In the wild-type strain we generally observed different patterns for the two inducers, sophorose and D-xylose. In both strains the differences on the TATA-box were found to be less pronounced on sophorose. In other words, the comparative in vivo footprinting patterns for sophorose for the wild-type strain became similar to those obtained for Rut-C30. For Rut-C30, it has previously been suggested that the $x y n 2$ URR is permanently bound by regulatory proteins and remains free of nucleosomes (Würleitner et al. 2003). 
According to our results, sophorose - other than D-xylose - leads to a similar DNA occupancy as D-glucose in both strains. Bearing in mind that i) the chromatin became as loose on sophorose as on D-xylose (compared to D-glucose), and ii) that the extent of the achieved xyn2 induction was also similar with both substances (Fig. $2 \mathrm{C}$ and D), we speculated that induction by sophorose is mediated without additional proteins binding. There are possible alternative mechanisms, which would fit to the similar in vivo footprinting pattern, e.g.: i) regulatory proteins that act in a carbohydrate-specific manner bind somewhere else in the respective URR, ii) chromatin remodelers that act in a carbohydrate-specific manner bind equally distributed on the URR (yielding the observed, regular pattern of small differences), iii) chromatin remodelers or histone modifying proteins bind in a carbohydrate-unspecific manner, but differ in their action (e.g. histone acetyltransferases bind and provoke an opening of chromatin under inducing conditions, while under repressing conditions histone methyltransferases bind and provoke a more close chromatin).

However, all these hypotheses would mean that the type of signal transmission in case of Dxylose and sophorose is strikingly different. Regarding sophorose, a cAMP-dependent signal transmission was shown to be involved in enzyme production in T. reesei (Šesták \& Farkaš 1992, Nogueira et al. 2015). Nogueria and co-workers found that the intracellular cAMP levels correlate directly with a higher induction level of cellulolytic enzyme expression in a sophorose-dependent manner (Nogueira et al. 2015). However, there does not exist such a study for the induction of xylanases in the presence of D-xylose or sophorose. Despite that, for Aspergillus phoeniciis and Aspergillus sydowii it was demonstrated that CCR of xylanase expression in the presence of Dglucose is similar as for xynl in T. reesei. The repression in Aspergillus could be reverted by the addition of exogenous cAMP (Gosh \& Nanda 1994, Rizzatti et al. 2008). Nonetheless the addition of exogenous cAMP did not revert the repression of cellulases on D-glucose in T. reesei (Šesták \& Farkaš 1992), one could speculate that there might be a cAMP-dependent release of CCR in the case of xylanases in $T$. reesei.

\section{Acknowledgements}

This work was financed by two grants from the Austrian Science Fund (FWF): V232-B20 and P24851 given to Astrid R. Mach-Aigner, and by a doctoral program of TU Wien ("CatMat").

\section{References}

Aro N, Ilmén M, Saloheimo A, Penttilä M. 2003 - ACEI of Trichoderma reesei is a repressor of cellulase and xylanase expression. Applied and environmental Microbiology 69, 56-65.

Aro N, Saloheimo A, Ilmén M, Penttilä M. 2001 - ACEII, a novel transcriptional activator involved in regulation of cellulase and xylanase genes of Trichoderma reesei. The Journal of biological chemistry 276, 24309-24314.

Derntl C, Gudynaite-Savitch L, Calixte S, White T, Mach RL, Mach-Aigner AR. 2013 - Mutation of the Xylanase regulator 1 causes a glucose blind hydrolase expressing phenotype in industrially used Trichoderma strains. Biotechnology for biofuels 6, 62.

Derntl C, Rassinger A, Srebotnik E, Mach RL, Mach-Aigner AR. 2015 - Xpp1 regulates expression of xylanases but not of cellulases in Trichoderma reesei. Biotechnology for biofuels 8, 11 .

Furukawa T, Shida Y, Kitagami N, Mori K, Kato M, Kobayashi T, Okada H, Ogasawara W, Morikawa Y. 2009 - Identification of specific binding sites for XYR1, a transcriptional activator of cellulolytic and xylanolytic genes in Trichoderma reesei. Fungal Genetics and Biology 46, 564-574.

Gorsche R, Jovanovic B, Gudynaite-Savitch L, Mach RL, Mach-Aigner AR. 2013 - A highly sensitive in vivo footprinting technique for condition-dependent identification of cis elements Nucleic Acids Research 42, e1. 
Gosh M, Nanda G. 1994 - Physiological studies on xylose induction and glucose repression of xylanolytic enzymes in Aspergillus sydowii MG49. FEBS Microbiology Letters 117, 151156.

Kuhls K, Leichfeldt E, Samules G, Kovacs W, Meyer W, Petrini O, Gams W, Börner T, Kubicek C. 1996 - Molecular evidence that the asexual industrial fungus Trichoderma reesei is a clonal derivative of the ascomycete Hypocrea jecorina. Proceedings of the National Academy of Science of the United States of America 93, 7755-7760.

Mach RL, Strauss J, Zeilinger S, Schindler M, Kubicek CP. 1996 - Carbon catabolite repression of xylanase I (xyn1) gene expression in Trichoderma reesei. Molecular Microbiology 21, $1273-1281$.

Mach-Aigner AR, Grosstessner-Hain K, Pocas-Fonseca MJ, Mechtler K, Mach RL. 2010 - From an electrophoretic mobility shift assay to isolated transcription factors: a fast genomicproteomic approach. BMC Genomics 11, 644.

Mandels M. 1985 - Applications of cellulases. Biochemical Society Transactions 13, 414-416.

Mello-de-Sousa TM, Gorsche R, Rassinger A, Pocas-Fonseca MJ, Mach RL, Mach-Aigner AR. 2014 - A truncated form of the Carbon catabolite repressor 1 increases cellulase production in Trichoderma reesei. Biotechnololy for Biofuels 7, 129.

Mello-de-Sousa TM, Rassinger A, Derntl C, Poças-Fonseca MJ, Mach-Aigner AR, Mach RL. 2016 - The Relation between chromatin status, Xyr1 and cellulase expression in Trichoderma reesei. Current Genomics 17, 1-8.

Mello-de-Sousa TM, Rassinger A, Pucher ME, dos Santos Castro L, Persinoti GF, Silva-Rocha R, Poças-Fonseca MJ, Mach RL, Silva RN, Mach-Aigner AR. 2015 - The impact of chromatin remodelling on cellulase expression in Trichoderma reesei. BMC Genomics 16.

Montenecourt BS, Eveleigh DE. 1979a - Production and characteriation of high yielding cellulase mutants of Trichoderma reesei. TAPPI Journal 28, 101-108.

Montenecourt BS, Eveleigh DE. 1979b. Selective Screening Methods for the Isolation of High Yielding Cellulase Mutants of Trichoderma reesei. In: Brown RD, Jurasek L, editors. Hydrolysis of Cellulose: Mechanisms of Enzymatic and Acid Catalysis. Washington, DC: American Chemical Society, 289-301.

Nogueira KMV, do Nascimento Costa M, de Paula RG, Mendonça-Natividade FC, Ricci-Azevedo R, Nascimento Silva. 2015 - Evidence of cAMP involvement in cellobiohydrolase expression and secretion by Trichoderma reesei in presence of the inducer sophorose. BMC Microbiology 15, 12.

Rauscher R, Würleitner E, Wacenovsky C, Aro N, Stricker AR, Zeilinger S, Kubicek CP, Penttilä M, Mach RL. 2006 - Transcriptional regulation of xynl, encoding xylanase I, in Hypocrea jecorina. Eukaryotic Cell 5, 447-456.

Ries L, Belshaw NJ, Ilmén M, Penttilä ME, Alapuranen M, Archer DB. 2014 - The role of CRE1 in nucleosome positioning within the $c b h 1$ promoter and coding regions of Trichoderma reesei. Applied Microbiology and Biotechnology 98, 749-762.

Rizzatti ACS, Freitas FZ, Bertolini MC, Peixoto-Nogueira SC, Terenzi HF, Jorge JA, Polizeli MLTM. 2008 - Regulation of xylanase in Aspergillus phoenicis: a physiological and molecular approach. Journal of Industrial Microbiology and Biotechnology 35, 237-244.

Šesták S, Farkaš V. 1992 - Metabolic regulation of endoglucanase synthesis in Trichoderma reesei: participation of cyclic AMP and glucose-6-phosphate. Canadian Journal of Microbiology 39, 342-347.

Steiger MG, Mach RL, Mach-Aigner AR. 2010 - An accurate normalization strategy for RT-qPCR in Hypocrea jecorina (Trichoderma reesei). Journal of Biotechnology 145, 30-37.

Strauss J, Mach RL, Zeilinger S, Hartler G, Stoffler G, Wolschek M, Kubicek CP. 1995 - Cre1, the carbon catabolite repressor protein from Trichoderma reesei. FEBS Letters 376, 103-107.

Stricker AR, Grosstessner-Hain K, Würleitner E, Mach RL. 2006 - Xyr1 (xylanase regulator 1) regulates both the hydrolytic enzyme system and D-xylose metabolism in Hypocrea jecorina. Eukaryotic Cell 5, 2128-2137. 
Stricker AR, Mach RL, de Graaff LH. 2008a - Regulation of transcription of cellulases- and hemicellulases-encoding genes in Aspergillus niger and Hypocrea jecorina (Trichoderma reesei). Applied Microbiology and Biotechnology 78, 211-220.

Stricker AR, Trefflinger P, Aro N, Penttilä M, Mach RL. 2008b - Role of Ace2 (Activator of Cellulases 2) within the xyn2 transcriptosome of Hypocrea jecorina. Fungal Genetics and Biology 45, 436-445.

Teeri T, Salovouri, I., Knowles, J. 1983 - The molecular cloning of the major cellulase gene from Trichoderma reesei. Biotechnology 1, 696-699.

Törrönen A, Mach RL, Messner R, Gonzalez R, Kalkkinen N, Harkki A, Kubicek CP. 1992 - The two major xylanases from Trichoderma reesei: characterization of both enzymes and genes. Biotechnology (N Y) 10, 1461-1465.

Viikari L, Kantelinen A, Sundquist J, Linko M. 1994 - Xylanases in bleaching - from an idea to the industry. FEMS Microbiology Reviews 13, 335-350.

Würleitner E, Pera L, Wacenovsky C, Cziferszky A, Zeilinger S, Kubicek CP, Mach RL. 2003 Transcriptional regulation of xyn2 in Hypocrea jecorina. Eukaryotic Cell 2, 150-158.

Zeilinger S, Mach RL, Schindler M, Herzog P, Kubicek CP. 1996 - Different inducibility of expression of the two xylanase genes xyn1 and xyn2 in Trichoderma reesei. Journal of Biological Chemistry 271, 25624-25629.

Zeilinger S, Schmoll M, Pail M, Mach RL, Kubicek CP. 2003 - Nucleosome transactions on the Hypocrea jecorina (Trichoderma reesei) cellulase promoter $c b h 2$ associated with cellulase induction. Molecular Genetics and Genomics 270, 46-55. 